\title{
Longitudinal Trajectories of Pneumonia Lesions and Lymphocyte counts Associated with Disease Severity among Convalescent COVID-19 Patients: A Group-based Multi-trajectory Analysis
}

\section{nannan shi}

Shanghai Public Health Clinical Center, Fudan University

chao huang

Shanghai Key Laboratory of Artificial Intelligence for Medical Image and Knowledge Graph, Shanghai qi zhang

Shanghai Key Laboratory of Artificial Intelligence for Medical Image and Knowledge Graph, Shanghai chunzi shi

Shanghai Public Health Clinical Center, Fudan University

fengjun liu

Shanghai Public Health Clinical Center, Fudan University

\section{fengxiang song}

Shanghai Public Health Clinical Center, Fudan University

qinguo hou

Shanghai Public Health Clinical Center, Fudan University

jie shen

Shanghai Public Health Clinical Center, Fudan University

fei shan

Shanghai Public Health Clinical Center, Fudan University

xiaoming su

Shanghai Key Laboratory of Artificial Intelligence for Medical Image and Knowledge Graph, Shanghai jia liu

Shanghai Key Laboratory of Artificial Intelligence for Medical Image and Knowledge Graph, Shanghai zhiyong zhang

Shanghai Public Health Clinical Center, Fudan University

lei shi

Shanghai Key Laboratory of Artificial Intelligence for Medical Image and Knowledge Graph, Shanghai yuxin shi ( $\sim$ shiyx828288@163.com)

Shanghai Public Health Clinical Center, Fudan University 


\section{Research Article}

Keywords: COVID-19, Group-based Multi-trajectory Modelling, Clinical Course, Pneumonia, Lymphocyte Posted Date: January 21st, 2021

DOl: https://doi.org/10.21203/rs.3.rs-139905/v1

License: (c) (i) This work is licensed under a Creative Commons Attribution 4.0 International License. Read Full License

Version of Record: A version of this preprint was published at BMC Pulmonary Medicine on July 13th, 2021. See the published version at https://doi.org/10.1186/s12890-021-01592-6. 


\section{Abstract}

\section{Background}

To explore the long-term trajectories considering pneumonia volumes and lymphocyte counts with individual data in COVID-19.

\section{Methods}

A cohort of 257 convalescent COVID-19 patients (131 male and 126 females) were included. Group-based multi-trajectory modelling was applied to identify different trajectories in terms of pneumonia lesion percentage and lymphocyte counts covering the time from onset to post-discharge follow-ups. We studied the basic characteristics and disease severity associated with the trajectories.

\section{Results}

We characterised four distinct trajectory subgroups. (1) Group 1 (13.9\%), pneumonia increased until a peak lesion percentage of $1.9 \%$ (IQR $0.7 \sim 4.4$ ) before absorption. The slightly decreased lymphocyte rapidly recovered to the top half of the normal range. (2) Group 2 (44.7\%), the peak lesion percentage was 7.2\% (IQR 3.2 12.7). The abnormal lymphocyte count restored to normal soon. (3) Group 3 (26.0\%), the peak lesion percentage reached $14.2 \%$ (IQR 8.5 19.8). The lymphocytes continuously dropped to $0.75 \times$ $10^{9} / \mathrm{L}$ after one day post-onset before slowly recovering. (4) Group $4(15.4 \%)$, the peak lesion percentage reached 41.4\% (IQR 34.8 47.9), much higher than other groups. Lymphopenia was aggravated until the lymphocytes declined to $0.80 \times 10^{9} / \mathrm{L}$ on the fourth day and slowly recovered later. Patients in the higher order groups were older and more likely to have hypertension and diabetes (all P values $<0.05$ ), and have more severe disease.

\section{Conclusions}

Our findings provide new insights to understand the heterogeneous natural courses of COVID-19 patients and the associations of distinct trajectories with disease severity, which is essential to improve the early risk assessment, patient monitoring, and follow-up schedule.

\section{Background}

The ongoing coronavirus disease 2019 (COVID-19) pandemic poses unprecedented challenges to healthcare systems worldwide. Medical scientists have claimed that it is urgent to understand the complete natural course of COVID-19 to optimise follow-up strategies to improve patient outcomes such as minimising persistent sequelae(1). To date, several related studies were published(2-7), which, however, were limited for at least two reasons. On one hand, only one data source, mainly pulmonary computed tomography (CT) or one immuno-related biomarker, was used to assess the disease course, thus cannot provide comprehensive information of COVID-19 which could involve multi-system impairment(8). On the other hand, the population-level averages of different internals over time were 
computed and connected to outline the course. Such approach mixed all patients together and could not differentiate the heterogeneity between patients who had distinct disease severity, clinical progression, and long-term prognosis(5, 9).

Group-based multi-trajectory modelling (GBMTM) is a latent class technique that takes full advantage of multivariate longitudinal data that are interrelated and complementary indicators of disease progression(10). GBMTM decomposes the population into distinct groups, each with a different underlying trajectory in terms of the studied biomarkers, therefore is suitable for capturing the longitudinal characteristics of COVID-19.

Pneumonia lesion volumes on CT and lymphocyte counts might compose a proper combination to track the COVID-19 disease course. First, these two biomarkers provide complementary information, and are both highly associated with disease severity and prognosis and are useful for diagnosis, triage, and monitoring of the disease $(11-14)$. Percentage of lesion volume is an intuitive metric to quantitatively assess the pneumonia severity(15). Severe pneumonia can lead to acute respiratory distress syndrome and increase mortality. Lymphocytes play an important role in maintaining the immune system function. Decreasing lymphocytes indicate that a number of immune cells are damaged and immune function is impaired. Second, both abnormal imaging results and decreased lymphocytes are typical characteristics commonly observed among COVID-19 patients and longitudinal tracing data could be available across the clinical course(16).

The primary goal of this study was applying GBMTM to identify distinct longitudinal trajectories in terms of pneumonia lesion percentage and lymphocyte count to efficiently and transparently assess the dynamic nature of the disease course starting from the symptom onset to post-discharge among various COVID-19 patients. The identified trajectories could be used to subtype patients for further studies and provide guidance for optimising follow-up schedules on a case-by-case basis.

\section{Methods}

\section{Ethics statement}

This retrospective study was approved by the Ethics Committee of Shanghai Public Health Clinical Center (SPHCC) (YJ-2020-S035-01) and was carried out in adherence with the Declaration of Helsinki. The need for informed consent from all patients was waived due to the study's retrospective nature.

\section{Patients}

SPHCC is the only designated hospital for COVID-19 in Shanghai, with the first case reported on January 20, 2020. By March 31, 2020, 328 laboratory-confirmed patients were discharged and were initially eligible for our research. Inclusion criteria were applied: (1) age $\geq 18$ years old; (2) reported history of symptoms and the date of initial symptoms; (3) with at least one post-discharge follow-up; (4) diagnosed 
as mild cases with no pneumonia lesions on CT scans throughout hospitalisation. As a result, a cohort of 257 COVID-19 patients (131 male and 126 females) were included for GBMTM trajectory analysis (Figure $1) \otimes$ and were followed up after discharge until June 1, 2020 with clinical symptoms monitored and blood tests and CT scans conducted as needed. For the sake of research integrity, we further investigated the mild $(\mathrm{N}=5)$ and fatal cases $(\mathrm{N}=7)$ for comparison.

\section{Data extraction}

Clinical data were obtained from the electronic medical record system, including sociodemographic characteristics (for example, age and sex), clinical characteristics (for example, the date of initial symptom onset, date of discharge, clinical subtype on admission, worst clinical subtype during hospitalisation, symptoms, and comorbidity) and longitudinal lymphocyte count records. Lymphopenia was defined as lymphocyte count below $1.1 \times 10^{9} / \mathrm{L}$. COVID-19 patients were categorized into mild, moderate, severe, and critical cases according to the Chinese national guidelines $(17,18)$. Chest CT acquisition and automatic COVID-19 pneumonia lesion quantification based on artificial intelligence are detailed in the supplementary materials.

\section{Group-based multi-trajectory modelling}

We applied GBMTM to identify subgroups of COVID-19 who had distinct longitudinal trajectories with respect to lesion percentage and lymphocyte count routinely collected from onset to post-discharge. Time 0 of the trajectory was the date of initial symptom onset. GBMTM analyses were introduced in detail in the supplementary materials.

\section{Statistical analysis}

Continuous data were expressed as median (interquartile range [IQR]), and categorical data were denoted as count (percentage). Data between trajectory groups were compared with the Kruskal-Wallis, chisquared, and Fisher's exact tests as appropriate. A two-tailed $P$ value less than 0.05 was considered statistically significant. All of the analyses were conducted using R software version 3.6.2 (R Foundation for Statistical Computing, Vienna, Austria).

\section{Results}

\section{Patient characteristics}

The median age of the 257 patients included in the GBMTM analysis was 51 years (IQR $37 \sim 63$ ), 131 were male $(51.0 \%)$, and 20 were smokers $(7.8 \%)$ (Table 1$)$. The most common symptoms were fever (83.3\%) and cough (81.7\%). Most patients were moderate cases on admission (97.3\%) and during 
hospitalisation (94.2\%). At admission, only 6 patients were severe or critical cases, but this number increased to 15 during hospitalisation. A total of 1,815 CT scans and 1,776 lymphocyte count records were collected. The median numbers of CT scans and lymphocyte data points per patient were 7 (IQR 6 8) and 6 (IQR $5 \sim 8$ ), respectively. Of note, we also analysed 5 mild cases and 7 fatal cases for comparison. 
Table 1

Clinical characteristics of the 257 included patients

\begin{tabular}{|c|c|}
\hline Characteristics & Value \\
\hline Age, years (IQR) & $51(37,63)$ \\
\hline Male sex, n (\%) & $131(51.0 \%)$ \\
\hline Smoking, n (\%) & $20(7.8 \%)$ \\
\hline \multicolumn{2}{|l|}{ Symptoms } \\
\hline Fever & $214(83.3 \%)$ \\
\hline Cough & $210(81.7 \%)$ \\
\hline Diarrhoea & $49(19.1 \%)$ \\
\hline \multicolumn{2}{|l|}{ Clinical subtype on admission } \\
\hline Mild & $1(0.4 \%)$ \\
\hline Moderate & $250(97.3 \%)$ \\
\hline Severe & $5(1.9 \%)$ \\
\hline Critical & $1(0.4 \%)$ \\
\hline \multicolumn{2}{|l|}{ Worst clinical subtype during hospitalisation } \\
\hline Moderate & $242(94.2 \%)$ \\
\hline Severe & $10(3.9 \%)$ \\
\hline Critical & $5(1.9 \%)$ \\
\hline \multicolumn{2}{|l|}{ Comorbidity } \\
\hline Cardiovascular disease & $18(7.0 \%)$ \\
\hline Hypertension & $59(23.0 \%)$ \\
\hline Coronary heart disease & $18(7.0 \%)$ \\
\hline Diabetes & $27(10.5 \%)$ \\
\hline Chronic obstructive pulmonary disease & $6(2.3 \%)$ \\
\hline Cancer & $6(2.3 \%)$ \\
\hline Number of CT scans per patient & $7(6,8)$ \\
\hline Number of lymphocyte count tests per patient & $6(5,8)$ \\
\hline
\end{tabular}




\section{Description of the trajectory groups}

The model most optimised for fit identified four distinct trajectory groups in terms of pneumonia lesions and lymphocyte counts (Fig. 2A). For lesion percentage, all the four trajectories followed a mountain-like shape of soaring to a peak and then being gradually absorbed. From Groups 1 to 4 , both the peak lesion percentage and time from onset to peak increased in steps, suggesting the increasing severity of pneumonia (Table 2). Two main types of lymphocyte count patterns existed. One started at a low level and then rapidly increased, while the other declined for a short time and then slowly recovered. Table S1 displays the metrics of adequacy and fit for the ultimate trajectory model, all demonstrating that the model was well fit.

Table 2

Characteristics of peak lesion percentages by trajectory group

\begin{tabular}{|c|c|c|c|c|c|c|}
\hline Variable & $\begin{array}{l}\text { Overall } \\
(\mathrm{N}= \\
257)\end{array}$ & $\begin{array}{l}\text { Group } \\
1 \\
(\mathrm{~N}= \\
36)\end{array}$ & $\begin{array}{l}\text { Group } 2 \\
(\mathrm{~N}= \\
117)\end{array}$ & $\begin{array}{l}\text { Group } 3 \\
(\mathrm{~N}=64)\end{array}$ & $\begin{array}{l}\text { Group } 4 \\
(\mathrm{~N}=40)\end{array}$ & $\begin{array}{l}P \\
\text { value }\end{array}$ \\
\hline Peak lesion percentage, $\%$ & $\begin{array}{l}9.3(3.9 \\
20.0)\end{array}$ & $\begin{array}{l}1.9 \\
(0.7 \\
4.4)\end{array}$ & $\begin{array}{l}7.2(3.2 \\
12.7)\end{array}$ & $\begin{array}{l}14.2 \\
(8.5, \\
19.8)\end{array}$ & $\begin{array}{l}41.4 \\
(34.8, \\
47.9)\end{array}$ & $\dot{0} 001$ \\
\hline $\begin{array}{l}\text { Days from symptom onset to } \\
\text { peak lesion percentage }\end{array}$ & $\begin{array}{l}10.0 \\
(7.0 \\
12.0)\end{array}$ & $\begin{array}{l}6.5 \\
(4.0, \\
9.2)\end{array}$ & $\begin{array}{l}9.0(6.0 \\
12.0)\end{array}$ & $\begin{array}{l}10.0 \\
(8.0, \\
12.0)\end{array}$ & $\begin{array}{l}12.0 \\
(10.8, \\
14.0)\end{array}$ & $\hat{0}_{0.001}$ \\
\hline
\end{tabular}

Note: Statistics presented: median (IQR). Statistical test performed: Kruskal-Wallis test.

Specifically, in Group 1, the pneumonia pattern was a small hill with the peak lesion percentage at 1.9\% (IQR $0.7 \sim 4.4$ ), and the time from onset to peak was 6.5 days (IQR $4.0 \sim 9.2$ ) (Fig. 2A, Table 2). No serious lymphopenia was observed before lymphocytes soon restored to $2 \times 10^{9} / \mathrm{L}$ and above after 4 days, indicating a rapid recovery of the immune system. A total of 36 patients (13.9\%) were assigned to Group 1.

In Group 2, the peak lesion percentage was 7.2\% (IQR 3.2 12.7), and the time from onset to peak was 9.0 days (IQR $6.0 \sim 12.0$ ). The lymphocyte count started from around $0.8 \times 10^{9} / \mathrm{L}$ and then increased to normal. The lymphocytes restored to $2 \times 10^{9} / \mathrm{L}$ after 63 days, indicating a fairly quick recovery of the immune function. A total of 115 patients (44.7\%) were assigned to Group 2.

In Group 3, the peak lesion percentage reached 14.2\% (IQR 8.5 19.8), and the time from onset to peak was 10.0 days (IQR $8.0 \sim 12.0$ ). Lymphopenia was exacerbated soon after onset as the lymphocyte count further decreased to $0.75 \times 10^{9} / \mathrm{L}$ after one day and then slowly recovered to normal. A total of 64 patients $(26.0 \%)$ were assigned to Group 3. 
In Group 4, the peak lesion percentage reached as high as 41.4\% (IQR 34.8 47.9), and the time from onset to peak was 12.0 days (IQR 10.8 14.0). The lymphocyte count declined for four days to a nadir of $0.80 \times 10^{9} / \mathrm{L}$ before gradually recovering. A total of 40 patients (15.4\%) were assigned to Group 4 . Both Groups 3 and 4 did not experience the repletion of lymphocytes to $2 \times 10^{9} / \mathrm{L}$ at the end of follow-up, which was as long as 80 days.

Among the four groups, there were significant differences in terms of the days from onset to discharge, the lesion percentages right before discharge, the lymphocyte counts right before discharge, and the percentage of patients who recovered from pneumonia (all $P<0.001$; Tables S2 and S3).

For the sake of missing enough data to assess the clinical course from admission to post-discharge, mild and fatal cases were not included in the GBMTM analysis. Instead, we manually plotted the trajectories of these two subpopulations for research integrity. The mild cases had zero lesion throughout hospitalisation, and their lymphocyte counts were maintained in the normal range of $1.1 \sim 3.2 \times 10^{9} / \mathrm{L}$ though fluctuated. As for the fatal cases, the lesion percentages increased rapidly to a much higher level than that of Group 4, while the lymphocyte count almost remained below $1 \times 10^{9} / \mathrm{L}$ (Fig. 2).

\section{Disease severity associated with trajectory group membership}

Clinical subtypes indicate overall disease severity. We further explored the associations between the trajectories and the clinical subtypes. For the clinical subtype on admission, patients in Group 1 were mostly moderate cases except one mild case (Table 3). All patients in Group 2 were moderate cases. Most patients in Group 3 were moderate cases but one patient was severe. Group 4 contained most severe or critical cases (83.3\%). Clinical subtypes could change as the patients recover or progress to be more severe, so we also investigated the worst clinical subtype during hospitalisation. During hospitalisation, the distribution of clinical subtypes for any of the first three trajectory groups remained fairly stable, except the prevalence of severe or critical cases in Group 4 increased from 12.5\% (5/40) to $32.5 \%(13 / 40)$. 
Table 3

Differences in disease severity between trajectory groups

\begin{tabular}{|c|c|c|c|c|c|}
\hline Characteristics & $\begin{array}{l}\text { Group } 1 \\
(\mathrm{~N}=36)\end{array}$ & $\begin{array}{l}\text { Group } 2 \\
(\mathrm{~N}=117)\end{array}$ & $\begin{array}{l}\text { Group } 3 \\
(\mathrm{~N}=64)\end{array}$ & $\begin{array}{l}\text { Group } 4 \\
(\mathrm{~N}=40)\end{array}$ & $\begin{array}{l}\mathrm{P} \\
\text { value }\end{array}$ \\
\hline Clinical subtype on admission & & & & & $<.001$ \\
\hline Mild & $1(2.8 \%)$ & $0(0 \%)$ & $0(0 \%)$ & $0(0 \%)$ & \\
\hline Moderate & $\begin{array}{l}35 \\
(97.2 \%)\end{array}$ & $\begin{array}{l}117 \\
(100 \%)\end{array}$ & $\begin{array}{l}63 \\
(98.4 \%)\end{array}$ & $\begin{array}{l}35 \\
(87.5 \%)\end{array}$ & \\
\hline Severe & $0(0 \%)$ & $0(0 \%)$ & $1(1.6 \%)$ & $\begin{array}{l}4 \\
(10.0 \%)\end{array}$ & \\
\hline Critical & $0(0 \%)$ & $0(0 \%)$ & $0(0 \%)$ & $1(2.5 \%)$ & \\
\hline \multicolumn{6}{|l|}{$\begin{array}{l}\text { Worst clinical subtype during } \\
\text { hospitalisation }\end{array}$} \\
\hline Moderate & $\begin{array}{l}36 \\
(100 \%)\end{array}$ & $\begin{array}{l}117 \\
(100 \%)\end{array}$ & $\begin{array}{l}62 \\
(96.9 \%)\end{array}$ & $\begin{array}{l}27 \\
(67.5 \%)\end{array}$ & \\
\hline Severe & $0(0 \%)$ & $0(0 \%)$ & $2(3.1 \%)$ & $\begin{array}{l}8 \\
(20.0 \%)\end{array}$ & \\
\hline Critical & $0(0 \%)$ & $0(0 \%)$ & $0(0 \%)$ & $\begin{array}{l}5 \\
(12.5 \%)\end{array}$ & \\
\hline
\end{tabular}

In short, from Groups 1 to 4, disease severity was increasingly worse, which was consistent from admission to discharge.

\section{Basic characteristics associated with trajectory group membership}

The basic characteristics investigated included age, sex, smoking history, and comorbidity (Table 4). Overall, age, hypertension and diabetes were significantly different between the groups. The median age was 34 years (IQR $30 \sim 41$ ), 44 years (IQR $36 \sim 60$ ), 56 years (IQR $46 \sim 64$ ), and 65 years (IQR $52 \sim 69$ ) in Groups 1 to 4 , respectively $(P<0.001)$. Patients in Group 1 were most unlikely to be hypertensive $(2.8 \%$ compared to higher than $20 \%$ in the other groups [P=0.013]). No patients in Group 1 had diabetes but the prevalence of diabetes in Group 4 was as high as $20 \%(P=0.021)$. 
Table 4

Basic characteristics by trajectory group

\begin{tabular}{|c|c|c|c|c|c|}
\hline Characteristics & $\begin{array}{l}\text { Group } 1 \\
(\mathrm{~N}=36)\end{array}$ & $\begin{array}{l}\text { Group } 2 \\
(\mathrm{~N}=117)\end{array}$ & $\begin{array}{l}\text { Group } 3 \\
(\mathrm{~N}=64)\end{array}$ & $\begin{array}{l}\text { Group } 4 \\
(\mathrm{~N}=40)\end{array}$ & $\begin{array}{l}P \\
\text { value }\end{array}$ \\
\hline Age, years (IQR) & $\begin{array}{l}34(30 \\
41)\end{array}$ & $\begin{array}{l}44(36 \\
60)\end{array}$ & $\begin{array}{l}56(46 \\
64)\end{array}$ & $\begin{array}{l}65(52, \\
69)\end{array}$ & $<.001$ \\
\hline Male sex, n (\%) & $\begin{array}{l}18 \\
(50.0 \%)\end{array}$ & $\begin{array}{l}59 \\
(50.4 \%)\end{array}$ & $\begin{array}{l}31 \\
(48.4 \%)\end{array}$ & $\begin{array}{l}23 \\
(57.5 \%)\end{array}$ & 0.832 \\
\hline Smoking, n (\%) & $4(11.1 \%)$ & $7(6.0 \%)$ & $5(7.8 \%)$ & $4(10.0 \%)$ & 0.630 \\
\hline \multicolumn{6}{|l|}{ Symptoms } \\
\hline Fever & $\begin{array}{l}28 \\
(77.8 \%)\end{array}$ & $\begin{array}{l}99 \\
(84.6 \%)\end{array}$ & $\begin{array}{l}50 \\
(78.1 \%)\end{array}$ & $\begin{array}{l}37 \\
(92.5 \%)\end{array}$ & 0.204 \\
\hline Cough & $\begin{array}{l}28 \\
(77.8 \%)\end{array}$ & $\begin{array}{l}94 \\
(80.3 \%)\end{array}$ & $\begin{array}{l}57 \\
(89.1 \%)\end{array}$ & $\begin{array}{l}31 \\
(77.5 \%)\end{array}$ & 0.346 \\
\hline Diarrhoea & $4(11.1 \%)$ & $\begin{array}{l}27 \\
(23.1 \%)\end{array}$ & $\begin{array}{l}11 \\
(17.2 \%)\end{array}$ & $7(17.5 \%)$ & 0.406 \\
\hline \multicolumn{6}{|l|}{ Comorbidity } \\
\hline Cardiovascular disease & $0(0 \%)$ & $9(7.7 \%)$ & $5(7.8 \%)$ & $4(10.0 \%)$ & 0.289 \\
\hline Hypertension & $1(2.8 \%)$ & $\begin{array}{l}30 \\
(25.6 \%)\end{array}$ & $\begin{array}{l}15 \\
(23.4 \%)\end{array}$ & $\begin{array}{l}13 \\
(32.5 \%)\end{array}$ & 0.013 \\
\hline Coronary heart disease & $0(0 \%)$ & $9(7.7 \%)$ & $5(7.8 \%)$ & $4(10.0 \%)$ & 0.289 \\
\hline Diabetes & $0(0 \%)$ & $\begin{array}{l}14 \\
(12.0 \%)\end{array}$ & $5(7.8 \%)$ & $8(20.0 \%)$ & 0.021 \\
\hline $\begin{array}{l}\text { Chronic obstructive pulmonary } \\
\text { disease }\end{array}$ & $0(0 \%)$ & $1(0.9 \%)$ & $4(6.2 \%)$ & $1(2.5 \%)$ & 0.100 \\
\hline Cancer & $2(5.6 \%)$ & $1(0.9 \%)$ & $3(4.7 \%)$ & $0(0 \%)$ & 0.108 \\
\hline
\end{tabular}

\section{Discussion}

Using GBMTM, for the first time we identified four distinct trajectory groups based on individual time series data of the long-term pneumonia lesion percentage and lymphocyte patterns, providing a comprehensive depiction of the COVID-19 disease course from admission to post-discharge. Regarding lesion percentage, all four trajectories appeared to be positively skewed hill-like curves, and the peak value progressively increased from Groups 1 to 4 . For lymphocyte counts, two main types were observed. Groups 1 and 2 started at a low level and then rapidly increased, while Groups 3 and 4 decreased for a 
short time and then slowly recovered. Patients in the higher order groups were older and more likely to have hypertension and diabetes. Patients in Groups 1 to 4 were characterised by sequentially more severe disease. Furthermore, we found fatal cases were worse than in Group 4 with significantly more pneumonia lesions and lower lymphocyte counts. In contrast, the mild cases demonstrated no pneumonia lesions and mostly normal lymphocyte counts.

Characterising the long-term trajectories enabled us to assess the population heterogeneity over the COVID-19 disease course. Despite the importance of longitudinal evaluation of chest CT and laboratory results as standards of clinical care, only simple and qualitative assessments of the follow-up reports were carried out subjectively in clinical practice. In research areas, a few studies explored the temporal changes of chest CT findings and reported that the lung abnormalities peaked $6-11$ days post onset(2, 6)'(19), which was consistent with our finding (Table 2). Chen et al. observed that decreased lymphocyte counts recovered to normal in convalescent patients, whereas they maintained low levels among the deceased(5). This finding was confirmed by our results. However, these previous studies ignored heterogeneity between patients by only estimating cross-sectional population-level summaries of only one biomarker in each analysis. For example, Wang et al. used the average proportion of ground-glass opacity at an interval of around 5 days(2). Instead, we took a step forward by applying GBMTM to analyse the patient-level time series of pneumonia lesion percentage and lymphocyte count, in which the interrelationships between the two biomarkers were also captured. Our results showed that COVID-19 patients were heterogeneous and the convalescent patients could be further divided into four subgroups.

Clustering COVID-19 patients into four trajectory groups could be a better method for understanding the disease severity of various patients and thus be useful for triage for tailored treatment. As shown in the results, the trajectory grouping was a stable indicator of overall disease severity, no matter compared to single measures such as lesion percentage, lymphocyte count, or clinical subtypes. This could be because the trajectory groups can capture the dynamic patterns of several key biomarkers instead of one single biomarker at only one single time point. As shown in Fig. 2A, from Groups 1 to 4, the peak lesion level and time from onset to peak level became increasingly larger, and the time for lymphocyte counts to rebound was gradually longer. The severe or critical cases were mostly in Groups 3 and 4 on admission, and more patients in Groups 3 and 4 developed more severe status during hospitalisation. These findings suggest that patients requiring a long time to resolve pneumonia and restore lymphocyte counts should be assigned to intensified care, especially those with declining lymphocyte counts.

Importantly, we observed that the lymphocyte counts were replenished much earlier than the resolution of lung lesions, indicating immune system restoration precedes lung tissue repair. Lymphocyte counts started to recover before symptom onset among the patients in Groups 1 and 2, and after 1 day and 4 days post-onset for the patients in Groups 3 and 4 . The durations for lymphocyte count recovery to the normal level (at least $1.1 \times 10^{9} / \mathrm{L}$ ) were 1 day, 2 days, 13 days, and 19 days in Groups 1 to 4, respectively. However, the durations before pneumonia began to resolve were $6.5,9,10$, and 12 days in Groups 1 to 4 , respectively. In light of these findings, monitoring the trends in lymphocyte alterations and the turning point of recovering lymphocytes may help rapidly identify patients at a high risk of adverse outcomes or 
who are recovering compared with pneumonia lesion volume changes on CT, which are organic injuries and will lag behind in reflecting the disease progression trend. As a result, optimised treatment could be promptly assigned to help reduce complications and mortality.

The heterogeneity in the longitudinal trajectories is also valuable for optimising post-discharge follow-up strategies, such as choosing proper follow-up intervals for different patients. The lesion percentages right before discharge were $0.6 \%, 2.7 \%, 6.0 \%$, and $24.6 \%$ in Groups 1 to 4 , and lymphocyte counts right before discharge were $2.5 \times 10^{9} / \mathrm{L}, 1.9 \times 10^{9} / \mathrm{L}, 1.2 \times 10^{9} / \mathrm{L}$, and $1.5 \times 10^{9} / \mathrm{L}$ in Groups 1 to 4 , respectively (Table S2). This demonstrated that the biomarkers were not fully recovered and the values varied across different COVID-19 patients at discharge, indicating that the follow-up strategy should consider such heterogeneity. For example, the follow-up interval after discharge might be chosen with time 0 as the day of symptom onset. Apart from Group 1, which always had a pneumonia percentage less than $5 \%$, the durations from onset to lesion percentage decreasing to $5 \%$ were estimated to be 26,45 , and 93 days in Groups 2 to 4 , respectively. The durations from onset to lymphocyte count returning to $1.1 \times 10^{9} / \mathrm{L}$ were estimated to be 1 day, 2 days, 13 days, and 19 days in Groups 1 to 4, respectively. Using these results, we can adjust the follow-up intervals of different subgroups. In general, patients in Groups 3 and 4 needed a more frequent follow-up plan to obtain more intensive surveillance during their longer convalescence period.

There are several limitations in our study. First, we focused on pneumonia lesion volume and lymphocyte counts for the trajectory analysis and ignored other clinical indicators, for example, leukocyte counts and D-dimers, which might have resulted in losing some pieces of the complete picture of COVID-19's disease evolution. However, CT imaging and lymphocyte counts are usually among the easiest biomarkers to access and interpret at most hospitals, thus making this study more reproducible. Second, to avoid the transmission risk caused by coughing and droplet formation during testing(20), no data on pulmonary function tests were collected during follow-up to assess the survivors' recovery of lung function. Third, the entire follow-up period was at least two months for all of the patients. Longer follow-up is expected to depict extended evolution of the disease. Finally, future studies are anticipated to collect more densified data points to predict the trajectory group membership of a patient in an early stage of the disease course, which would be beneficial to guide therapeutic strategies as a patient predicted in a higher order group should undergo more intensive surveillance to prevent undesirable outcomes.

\section{Conclusions}

In conclusion, our study identified four different trajectory groups in terms of pneumonia lesions quantified on CT and lymphocyte counts, an indicator of immune system function, in a cohort of convalescent COVID-19 patients with different characteristics between groups. Generally, in trajectory Groups 1 to 4 , the degree of disease severity gradually increased. These distinct trajectory groups suggested that COVID-19 patients are heterogenous and should be further subtyped and treated differently. These findings shed new light on understanding the underexplored course of the disease, highlight the need for clinicians to closely monitor the disease course even after hospital discharge, and 
provide new perspectives to study COVID-19 patients to generate knowledge for improved prevention, control, and therapeutics.

\section{Abbreviations}

COVID-19 = coronavirus disease 2019; CT = computed tomography; GBMTM = group-based multitrajectory modelling; SPHCC: Shanghai Public Health Clinical Center; IQR = interquartile range.

\section{Declarations}

\section{Ethics approval and consent to participate}

This retrospective study was approved by the Ethics Committee of Shanghai Public Health Clinical Center (SPHCC) (YJ-2020-S035-01) and was carried out in adherence with the Declaration of Helsinki. The need for informed consent from all patients was waived due to the study's retrospective nature.

\section{Consent for publication}

Not applicable.

\section{Availability of data and materials}

The datasets used and/or analysed during the current study are not publicly available as they contain identifiable and personal information of COVID-19 but are available from the corresponding author on reasonable request.

\section{Competing interests}

The authors declare that they have no competing interests.

\section{Funding}

This study has received funding by the Science and Technology Commission of Shanghai Municipality (No. 20441900600).

\section{Authors' contributions}

Conception and design: NS, QZ, CH, LS, and YS 
Administrative support: YS, LS, FS, XS, JL, and ZZ

Provision of study materials or patients: NS, CS, FL, FS, QH, and JS

Collection and assembly of data: NS, CS, FL, FS, QH, and JS

Data analysis and interpretation: $\mathrm{CH}, \mathrm{QZ}$, and NS

Manuscript writing: All authors

Final approval of manuscript: All authors

\section{Acknowledgements}

Not applicable.

\section{References}

1. Raghu G, Wilson KC. COVID-19 interstitial pneumonia: Monitoring the clinical course in survivors. Lancet Respir Med 2020

2. Wang Y, Dong C, Hu Y, Li C, Ren Q, Zhang X et al. Temporal changes of CT findings in 90 patients with COVID-19 pneumonia: A longitudinal study. Radiology 2020;296:E55-E64

3. Chung M, Bernheim A, Mei X, Zhang N, Huang M, Zeng X et al. CT Imaging Features of 2019 Novel Coronavirus (2019-nCoV). Radiology 2020;295:202-207

4. Rodrigues J, Hare SS, Edey A, Devaraj A, Jacob J, Johnstone A et al. An update on COVID-19 for the radiologist - a British society of Thoracic Imaging statement. Clin Radiol 2020;75:323-325

5. Chen R, Sang L, Jiang M, Yang Z, Jia N, Fu W et al. Longitudinal hematologic and immunologic variations associated with the progression of COVID-19 patients in China. J Allergy Clin Immunol 2020;146:89-100

6. Pan F, Ye T, Sun P, Gui S, Liang B, Li L et al. Time Course of Lung Changes at Chest CT during Recovery from Coronavirus Disease 2019 (COVID-19). Radiology 2020;295:715-721

7. Song F, Shi N, Shan F, Zhang Z, Shen J, Lu H et al. Emerging 2019 Novel Coronavirus (2019-nCoV) Pneumonia. Radiology 2020;295:210-217

8. Wang T, Du Z, Zhu F, Cao Z, An Y, Gao Y et al. Comorbidities and multi-organ injuries in the treatment of COVID-19. Lancet 2020;395:e52

9. Guan W, Ni Z, Hu Y, Liang W, Ou C, He J, et al. Clinical Characteristics of Coronavirus Disease 2019 in China. N Engl J Med. 2020;382(18):1708-1720.

10. Nagin DS, Jones BL, Passos VL, Tremblay RE. Group-based multi-trajectory modeling. Stat Methods Med Res 2018;27:2015-2023 
11. Dong $D$, Tang Z, Wang S, Hui H, Gong L, Lu Y et al. The role of imaging in the detection and management of COVID-19: A review. IEEE Rev Biomed Eng 2020;PP

12. Henry BM. COVID-19, ECMO, and lymphopenia: A word of caution. Lancet Respir Med 2020;8:e24

13. Shen Y, Zheng F, Sun D, Ling Y, Chen J, Li F et al. Epidemiology and clinical course of COVID-19 in Shanghai, China. Emerg Microbes Infect 2020;9:1537-1545

14. Ruan Q, Yang K, Wang W, Jiang L, Song J. Clinical predictors of mortality due to COVID-19 based on an analysis of data of 150 patients from Wuhan, China. Intensive Care Med 2020;46:846-848

15. Shen C, Yu N, Cai S, Zhou J, Sheng J, Liu K et al. Quantitative computed tomography analysis for stratifying the severity of Coronavirus Disease 2019. J Pharm Anal 2020

16. Hope MD, Raptis CA, Henry TS. Chest computed tomography for detection of coronavirus disease 2019 (COVID-19): Don't rush the science. Ann Intern Med 2020;173:147-148

17. National Health Commission of the People's Republic of China. National COVID-19 diagnosis and treatment scheme (the seventh edition). [cited 2020 Mar 3]. Available at:

www.nhc.gov.cn/yzygj/s7653p/202003/46c9294a7dfe4cef80dc7f5912eb1989.shtml. Accessed August 10, 2020.

18. Feng Y, Ling Y, Bai T, Xie Y, Huang J, Li J et al. COVID-19 with different severities: A multicenter study of clinical features. Am J Respir Crit Care Med 2020;201:1380-1388

19. Wang C, Huang P, Wang L, Zhujing S, Lin B, Wang Q et al. Temporal changes of COVID-19 pneumonia by mass evaluation using CT: A retrospective multi-center study. Annals of Translational Medicine $2020 ; 8$

20. Matucci-Cerinic M, Bruni C, Allanore Y, Clementi M, Dagna L, Damjanov NS et al. Systemic sclerosis and the COVID-19 pandemic: World Scleroderma Foundation preliminary advice for patient management. Ann Rheum Dis 2020;79:724-726

\section{Figures}




\section{COVID-19 patients discharged}

from January 25 to March 31, $2020(\mathrm{~N}=328)$

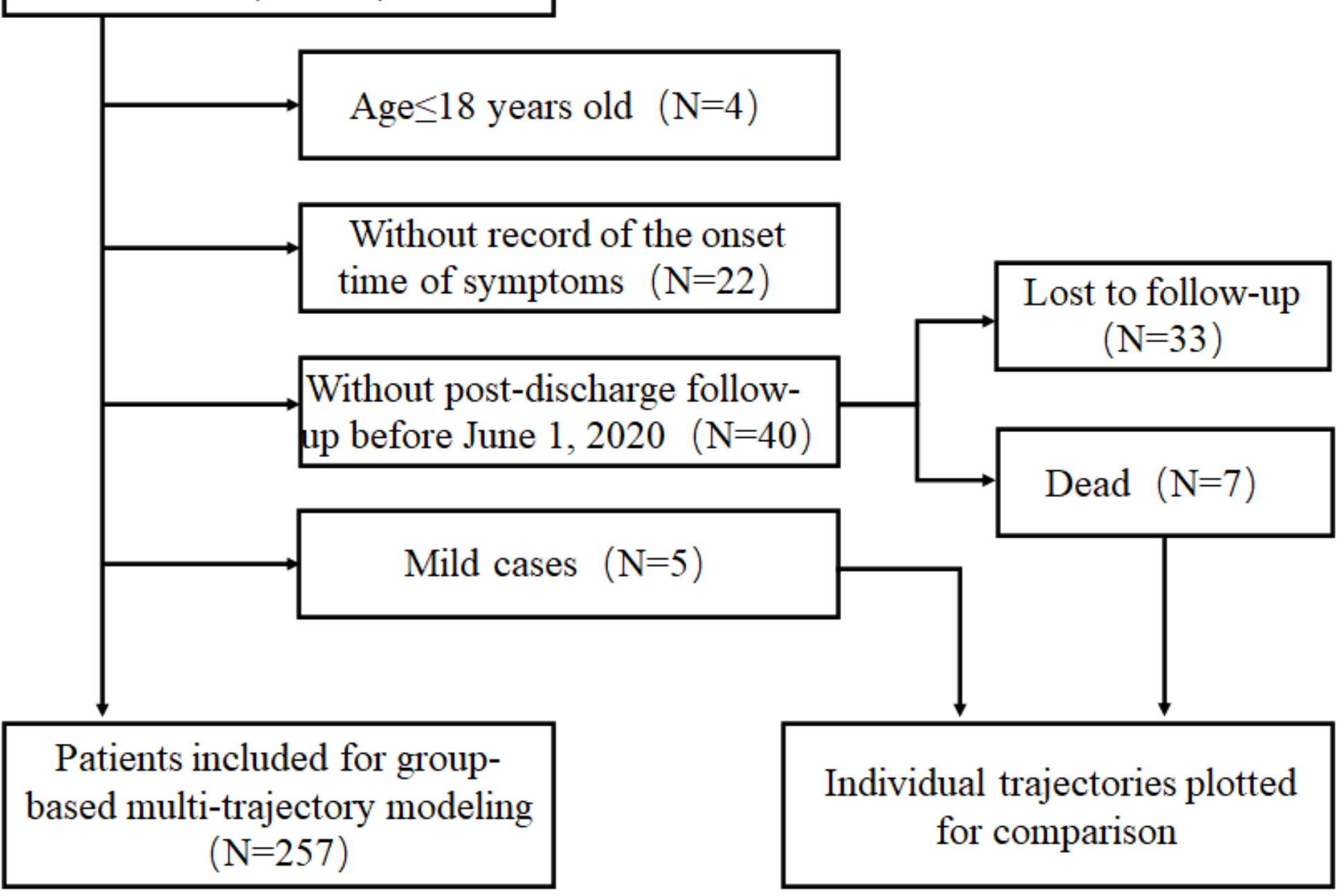

Figure 1

Patient selection flowchart 

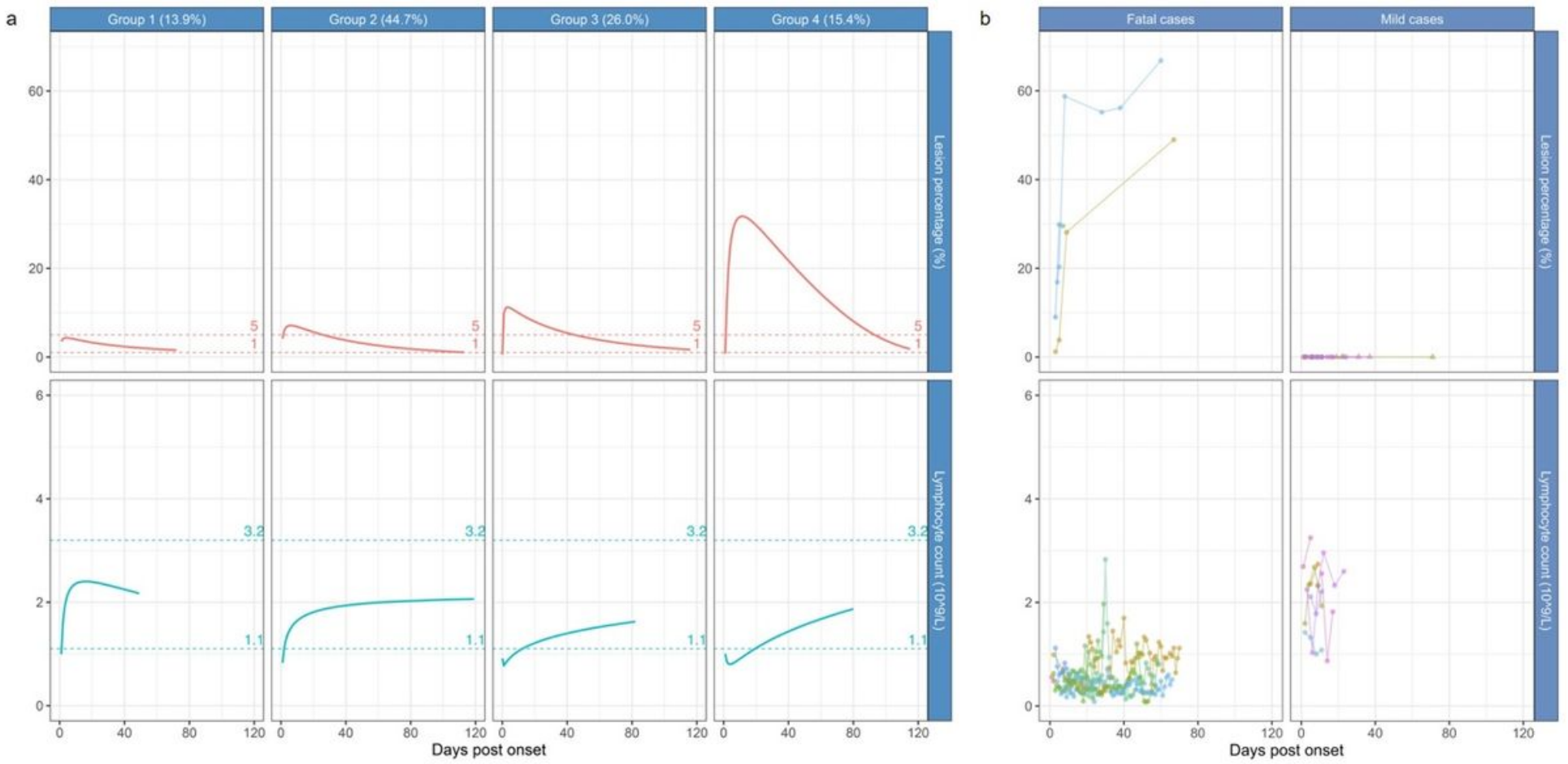

Figure 2

Longitudinal trajectories of lesion percentage (top row) and lymphocyte count (bottom row) among convalescent $(N=257)$, mild $(N=5)$, and dead $(N=7)$ COVID-19 patients. a) Estimated longitudinal trajectories for convalescent patients that were categorised into four groups based on group-based multitrajectory analysis. b) Individual longitudinal trajectories for mild and fatal cases with the observed data

\section{Supplementary Files}

This is a list of supplementary files associated with this preprint. Click to download.

- 0112SupplementaryMaterial.docx 